\title{
Business Agent
}

\author{
I.-Heng Meng ${ }^{1}$, Wei-Pang Yang ${ }^{1}$, Wen-Chih Chen ${ }^{2}$, Lu-Ping Chang ${ }^{2}$ \\ 1 Institute of Computer and Information Science., \\ National Chiao-Tung University, Hsinchu, Taiwan, R.O.C \\ 2 Advanced e-Commerce Technology Lab., Institute for Information Industry, ROC \\ wichenaiii.org.tw, clpaiii.org.tw
}

\begin{abstract}
The Internet and World Wide Web represent an increasingly important channel for retail commerce as well as business transactions. However, there are almost 5 billion pages or sites on the Internet and WWW. There lacks an integrated mediator business agent which is around the internet to connect between suppliers and users. Therefore, an intelligent business broking agent between supply and demand is needed for using and sharing the information efficiently and effectively. In this paper we proposed a new business agent architecture. The Business Spy Agent (BSA) captures the supply and demand information from e-commence sites automatically. Supply and Demand Analysis Mechanism (SDAM) uses NLP technologies to extract product and trading information. Domain ontology is used to classify between supply and demand and to divide them into subsets. A benefit model is proposed to handle the pairing between sub-supply and sub-demand. And a divide-and-conquer algorithm is proposed to handle the whole matching between supply and demand. In the negotiation process, the architecture uses NLP and template to produce negotiation text to handle negotiation through mail. Finally, Hidden Business Mining Mechanism (HBMM) adopts data mining technology to achieve hidden business mining. The architecture covers the four process of buying behavior including support and need identification, Product brokering, Merchant brokering, Negotiation.
\end{abstract}

\section{Introduction}

WWW had become the successful Platform for message exchange. Users can search and exchange messages through the Internet and WWW. Therefore, more and more trading have been done on the internet and WWW. Recently, there are almost 5 billion pages or sites on the Internet and WWW. Because of drowning in information, users or companies wasted lots times on searching and browsing huge number of sites to get the information of products and satisfy the demands. There lacks a integrated mediator business agent in sites which is around the internet, to connect the suppliers and users. Therefore, an intelligent business broking agent between supply and demand is needed for using and sharing the information efficiently and effectively. 


\section{Related work}

Up to now, most of business agents in the Internet support the services which like electronic market. In these service sites, the supporters can use the "Posting System" which is supported by the service site to publish the products information. Users can retrieve the products information through retrieval system provided by sites. Some sites provide the electronic prices quotation system which let users require price of products on sites. The XML technology is used for information exchanging makes the buying process electrolyzed. The sites including http://www.personalogic.com, http://www.firefly.com, http://jango.excite.com, http://www.onsale.com, http://www.ebary.com/aw,

http://auction.eecs.imich.edu, $\mathrm{http} / / /$ ecommerce.media.mit.edu/tete-a-tete/ and http://bf.cstar.ac.com/bfare are famous. These business agents usually provide electronic marketing sites which contain all business transactions. In other words, from products information to buying behaviors are all in this sites and can't across the sites. The sites can't help users to find the products and help supplier to find the hidden business. When the user has demands, he can use the category or search interface provided by site to retrieve the products. After finding the products, user can do compare the price, purchase the products, and entering payment information. Many descriptive theories and models sought to capture buying behavior, including the Andreasen model, the Bettman information-processing model, Nicosia model. All share six similar fundamental stages of the buying process [6]:

(1)Supply and Demand Identification. (2)Product Brokering. (3)Merchant Brokering. (4)Negotiation. (5)Purchase and Delivery. (6)Production Service and Evaluation.

The Purchase and Delivery process and Production Service and Evaluation process can't be completed by business agent. But others process can be done by business agent technologies. Most of the business agents didn't handle the Need Identification and only the T@T handles the processes including Product Brokering, Merchant Brokering and Negotiation[4]. We hope business agent is more intelligence. It can identify the real need of user through the huge product information in internet. Moreover, it can help user handle the processes including the Supply and Demand Identification process, the Product Brokering process, the Merchant Brokering process and the Negotiation process.

For the users, business agent could help user search needed products, compare price, handle negotiation and purchase. The things user must handle by self are payment and get the products. For supplier, business agent can help supplier find the hidden business. In this paper we propose a new architecture to construct business agent which use the NLP(Natural Language Process) and knowledge retrieval technologies to match supply and demand intelligently. 


\section{Business Agent}

\subsection{System architecture}

In the architecture, business agent contain 5 mechanism as shown in figure 1: (1)Business Spy Agent (BSA) - The supply and demand information is captured in electronic market site automatically. The supply and demand information could be analyzed in next mechanism to construct supply and demand list. (2)Supply and Demand Analysis Mechanism (SDAM) - Using NLP to analyze the information which is aggregated by Business Spy Agent and construct the supply and demand list. (3)Supply and Demand Clustering Mechanism (SDCM) - Using the clustering algorithm to cluster the information which is analyzed by previous mechanism. The clustering algorithm is based on the domain ontology [1]. (4) Matching and Negotiation Mechanism (MNM) - uses a benefit model and a matching algorithm to match supply and demand. (5) Hidden Business Mining Mechanism (HBMM) Using the data mining algorithm to mine the hidden business from business trading.

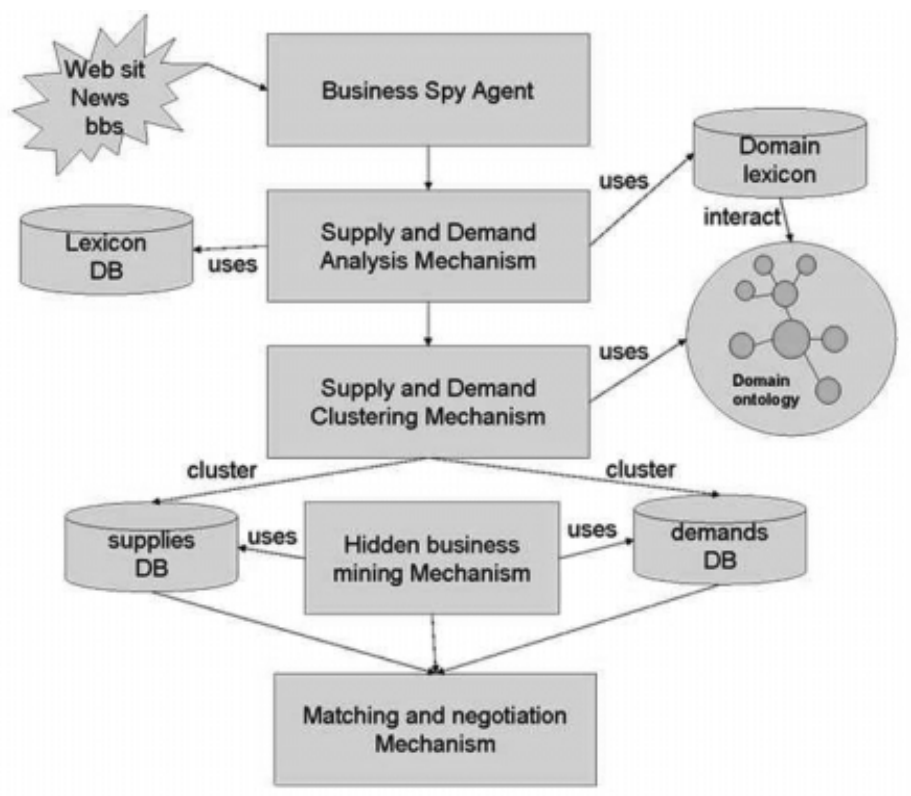

Fig. 1. System architecture

\subsection{Business spy agent}

There are many activities when a trading is made in internet. First, the supply (for suppler) and demand (for buyer) messages are posted. Then user waits for someone 
response destructively or does more posting and search activities. It is time-consuming processing to finish the business trading. In our architecture, the BSA (business spy agent) is used to capture the supply and demand from internet [3]. In order to complete the job, the knowledge base is used to store the famous electronic markets' URL (http://www.bid.com.tw), BBS (bbs.ntu.edu.tw) and news, etc. These sites have many supply and demand information which can be captured by business spy agent.

\subsection{Supply and Demand Analysis Mechanism}

The SDAM analyzes the information which is captured by BSA, and filters the redundancy and useless information. After filtering, the useful supplies and useful needs information are formatted. For example, the supply and demand are some simply free word sentences in Bulletin Board System (BBS). As shown in figure 2 and figure 3:

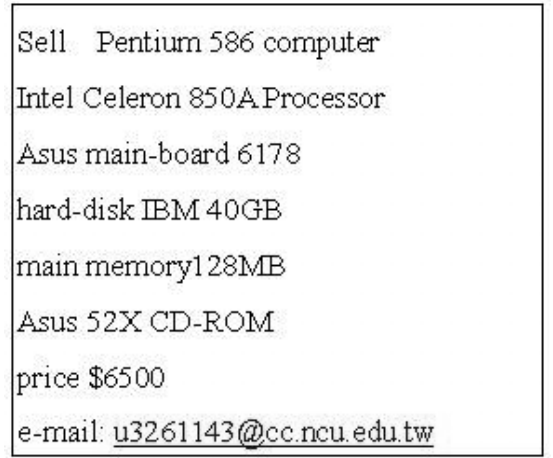

Fig. 2. Supply information

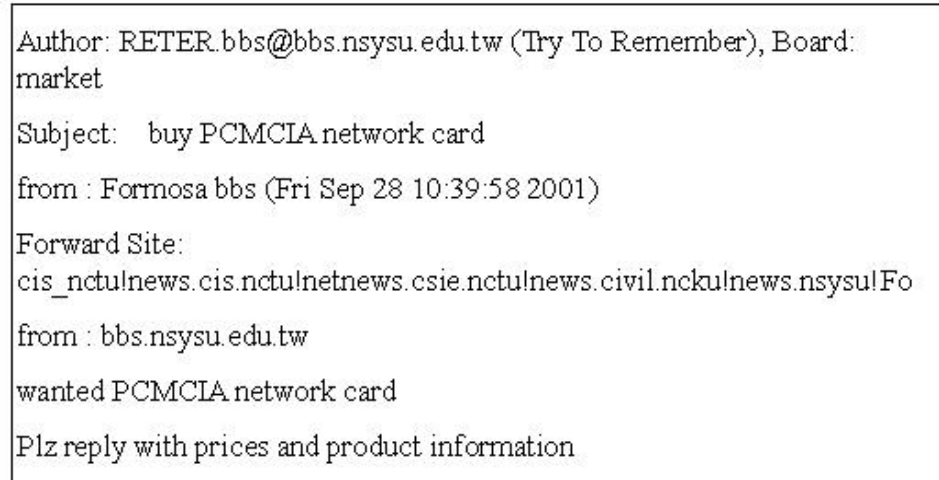

Fig. 3. Demand information

The SDAM uses the NLP technology to analyze the information and construct useful supply information" and "demand information". The analysis processing 
contains three steps:

(1) Supply and demand detection: The purpose of this step is to separate the supply and demand by using corpus which contain concept of trading. For example, "sell", "buy”, "wanted", “ask for"... etc. Using NLP technology, the supply and demand information can be separated by this way.

(2) Product information detection: After separate supply or demand information, the product information is detected. In this paper, we focus on computer hardware peripherals. We construct the domain ontology of computer hardware peripheral then using the ontology and NLP technology to extract the production information. The domain ontology and production can used in SDCM latter to achieve matching.

(3) Transaction information detection: The transaction information is detected in this step. The information contains delivery time, place, contact information ... etc.

\subsection{Supply and Demand Clustering Mechanism}

After the supply and demand information is detected through SDAM, the information can be clustered by SDCM. The purpose of clustering is to speed up matching supply and demand. The supply and demand belong to the same cluster are matched to finish supply and demand matching. In this paper, we use the ontology based clustering algorithm to cluster supply and demand. Each node in ontology considered as a cluster, Supply information and demand information are mapped to some nodes on ontology as shown in figure 4.
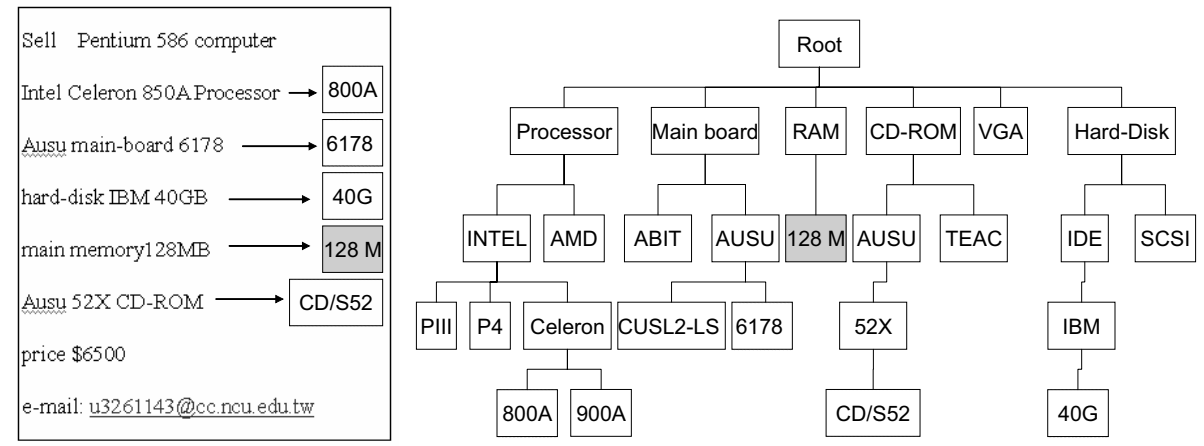

Fig. 4. Supply and demand mapping to nodes on ontology

The other advance of the clustering algorithm is that supplies and demands can be divided into sub-supplies and sub-demands. For example, the demand for computer can be divided to sub-demands including processor, main-board, hard-disk, main-memory...etc. When matching supply and demand, these sub-demands can be fitted by some sub-supplies and the whole matching can be achieved. 


\subsection{Matching and Negotiation Mechanism}

After clustering, supplies and demands are matched in this step. We describe the three types of supplies and demands matching and propose a Divide and Conquer Matching algorithm to achieve the work.

Type1: In this type just on supply can satisfy demand (one to one matching) as shown in figure 5. This type is the simplest type of matching and we just match the supply and demand. Then we can do the negotiation.

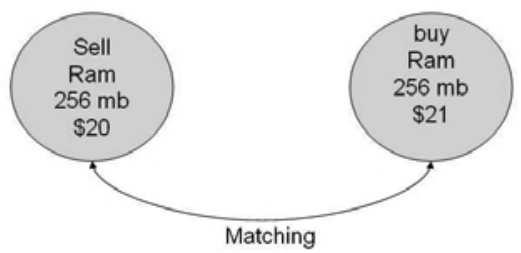

Fig. 5. One to one matching

Type2: In this type many supplies can satisfy demand (many to one matching) as shown in figure 6 . The best supply is selected to match for demand.

\begin{tabular}{|c|c|c|}
\hline Seller & Item & price \\
\hline A & $256 \mathrm{mb}$ ram & 20 \\
\hline$B$ & $256 \mathrm{mb}$ ram & 21 \\
\hline $\mathrm{C}$ & $256 \mathrm{mb}$ ram & 19.5 \\
\hline D & $256 \mathrm{mb}$ ram & 20 \\
\hline$E$ & $256 \mathrm{mb}$ ram & 22 \\
\hline
\end{tabular}

Fig. 6. Many to one matching

The benefit model which can select the best supply is based on price. The lower price of total sub-demand, the high benefit gets. The benefit model is:

$B_{i}= \begin{cases}\operatorname{Min}\left(\sum_{j \text { csub-demandof } i} B_{i j}, \text { price of supply pair of } D_{i}\right) & \text { if } D_{i} \text { can decomposeto sub-demands } D_{i j} \\ \text { price of supply pair of } D_{i} & \text {,if } D_{i} \text { can't decomposeto sub-demands }\end{cases}$

where $B_{i}$ is the benefit of demand $D_{i}$ and $B_{i j}$ is the benefit of sub-demand $D_{i j}$ of demand $D_{i}$.

Type3: Different supplies must be combined to satisfy demand in this type as show in figure7. Demand must be decomposed to sub-demands. For each sub-demand, a supply is matched to satisfy it or decompose it to more sub-demands. We propose a Divide and Conquer Matching algorithm to achieve the goal. The algorithm is shown in figure 8 . 


\begin{tabular}{|l|l|l|}
\hline seller & item & specification \\
\hline B & Memory & $256 \mathrm{mb}$ \\
\hline A & CPU & P-3 $800 \mathrm{hz}$ \\
\hline B & HD & $30 \mathrm{G}$ \\
\hline
\end{tabular}

\begin{tabular}{|c|c|c|}
\hline & Item & specification \\
\hline & CPU & P-3 700 above \\
\hline \multirow{2}{*}{ Matching } & Memory & $256 \mathrm{mb}$ \\
\hline & HD & $30 \mathrm{G}$ above \\
\hline
\end{tabular}

Fig. 7. Different supplies must be combined to satisfy demand

Function $\operatorname{Match}\left(D_{0}\right)$

For demand $D_{0}$ do begin

If $D_{0}$ can be divided into sub-demands according to domain ontology then

Divide $D_{0}$ into sub-demands $\left\{D_{01}, D_{02}, \ldots, D_{0 k}\right\}$

$B_{0}=0$

For each sub-demands $D_{0 k}$ do begin

$$
\begin{aligned}
& B_{0 k}=\operatorname{Match}\left(D_{0 k}\right) \\
& B_{0}=B_{0}+B_{0 k}
\end{aligned}
$$

End For

End If

If $D_{0}$ can be matched with supply without divided $D_{0}$ then

$C_{0}=$ price of matching supply with $D_{0}$

Else

$$
C_{0}=0
$$

End If

End For

If $C_{0}<>0$ then

$\operatorname{Return} \operatorname{Min}\left(B_{0}, C_{0}\right)$

Else

Return $B_{0}$

End If

End Function

Fig. 8. Divide and conquer algorithm for supply and demand matching

After supplies and demands are matched, the negotiation [2] must be handled. In our architecture, we provide hiding negotiation mechanism and use auto-mail technology to achieve negotiation. The supply and demand information in internet (WWW, BBS, News... etc.) contain some static information (product, price, time, place ... etc). The buy and sell templates are constructed previously. The negotiation mail is constructed by using NLP and select suitable template. Then the negotiation mechanism sends negotiation mails to suppler and user for achieving negotiation. The buying template is showed in figure 9 . 


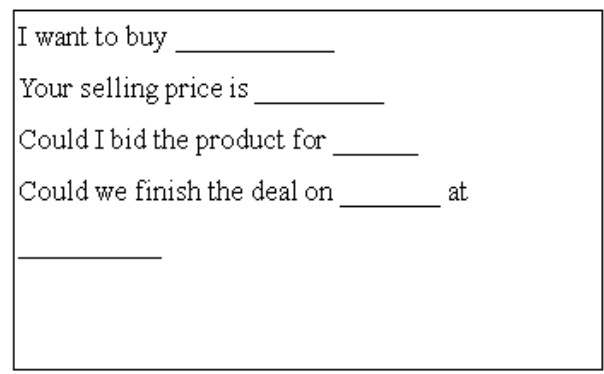

(a)

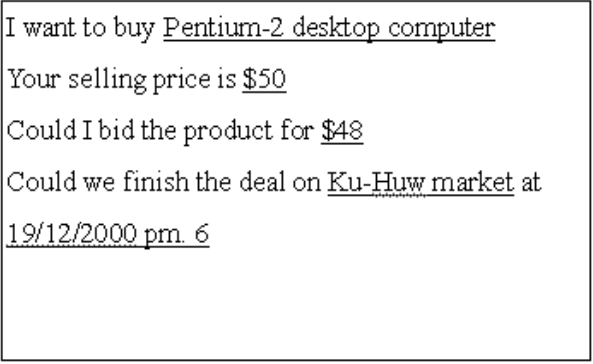

(b)

Fig. 9. (a) buying template (b) buying negotiation text

\subsection{Hidden business mining (HBM) mechanism}

In previous section, the negotiation is processed when there are demands. Sometimes, supply products need wait long time for sell or can't sell forever. In our architecture, The HBM (Hidden Business Mining) mechanism is used. The HBMM uses data mining technology to mining the hidden business and doing promotion. The association rules algorithm is used to achieve the goal [7]. The HBM mines the association rules by using history transaction log. If there is a rule "buy printer $=>$ buy printer paper", then the system retrieves the uses which had buy printer without printer paper. And the system does promotion of printer paper. The HBMM makes the process of business more complete.

\section{Conclusion and Future work}

In this paper, we propose a business agent architecture which covers the four process of buying behavior (Support and Demand Identification, Product Brokering, Merchant Brokering, Negotiation). The BSA captures the supply and demand information from e-commence sites automatically. SDAM uses NLP technologies to extract product and transaction information. Domain ontology is used to classify supply and demand and divide them into subsets. A benefit model is proposed to handle the matching between sub-supply and sub-demand. And a divide and conquer 
algorithm is proposed to handle the whole matching between supply and demand. In the negotiation process, the architecture uses NLP and template to product negotiation text and mailed through mail mechanism to handle negotiation. Finally, HBMM adopts data mining technology to achieve hidden business mining.

Acknowledgements

This research was supported by the III Innovative and Prospective Technologies Project of Institute for Information Industry and sponsored by MOEA, ROC

\section{References}

1. Bergamaschi, S., et al., "An Intelligent Approach to Information Integration," Guarino, N. (ed.) Formal Ontology in Information Systems. IOS Press 1998.

2. Chavez, A., Dreilinger, D., Guttman, R., and Maes, P. A real-life exper-iment in creating an agent marketplace. In Proceedings of the Second Inter-national Conference on the Practical Application of Intelligent Agents and Multi-Agent Technology PAAM'97 (London, U.K., Apr.). Practical Application Company, London, 1997.

3. Doorenbos, R., Etzioni, O., and Weld, D. A scalable comparison-shop-ping agent for the World-Wide Web. In Proceedings of the First Interna-tional Conference on Autonomous Agents Agents'97 (Marina del Rey, Calif., Feb. 5-8). ACM Press, N.Y., 1997, pp. 39-48

4. Guttman, R., and Maes, P. Agent-mediated integrative negotiation for retail electronic commerce. In Proceedings of the Workshop on Agent-Medi-ated Electronic Trading AMET'98 (Minneapolis, May 1998).

5. Moukas, A., Guttman, R., and Maes, P. Agent-mediated electronic com-merce: An MIT Media Laboratory perspective. In Proceedings of the Inter-national Conference on Electronic Commerce (Seoul, Korea, Apr. 6-9). ICEC, Seoul, 1998, pp. 9-15.

6. Pattie Maes, Robert H. Guttman, AND Alexandros G. Moukas. Ahents That Buy and Sell. COMMUNICATIONS OF THE ACM March 1999/vol. 42, No. 3.

7. R. Srikant, Q.Vu, and R. Agrawal. Mining association rules with item constraints. In Proc. 1997 Int. Conf. Knowledge Discovery and Data Mining (KDD’97), Newport Beach, CA, Aug.1997. 